\title{
Nice bases for primary Abelian groups
}

\section{Peter V. Danchev}

Published online: 13 November 2007

(C) Università degli Studi di Ferrara 2007

\section{Erratum to: Ann Univ Ferrara (2007) 53(1):39-50 DOI 10.1007/s11565-007-0004-2}

The original version of this article unfortunately contained a misprint. In page 49, line 23 , replace "> by " $\Rightarrow$ ".

The online version of the original article can be found under doi:10.1007/s11565-007-0004-2. 